\title{
ON THE CONVERGENCE OF THE FINITE ELEMENT APPROXIMATION OF EIGENFREQUENCIES AND EIGENVECTORS TO MAXWELL'S BOUNDARY VALUE PROBLEM
}

\author{
PEKKA NEITTAANMÄKI and RAINER PICARD
}

\section{Introduction}

This paper can be regarded as a supplementary to the work [10]. There the finite element approximation of the time-harmonic Maxwell's equations

$$
\left\{\begin{array}{l}
\operatorname{curl} E-i \omega \mu H=J \\
\operatorname{curl}_{*} H+i \omega \varepsilon E=K
\end{array} \text { in } G \subset \boldsymbol{R}^{2}\right.
$$

with homogeneous boundary condition

$$
n \wedge E_{\mid \Gamma}=0,
$$

$\Gamma:=\partial G, n \wedge E:=n_{1} E_{2}-n_{2} E_{1}$, was considered under the assumption that $\omega$ is not an eigenvalue of the system. Here $G$ is a bounded smooth domain in the plane, $n$ denotes the outer unit normal on the boundary $\Gamma, E$ and $H$ are vector and scalar functions and the operators curl and $\operatorname{curl}_{*}$ are in two space dimensions formally given by curl $E=\partial_{1} E_{2}-\partial_{2} E_{1}, \operatorname{curl}_{*} H=\left(\partial_{2} H \mid-\partial_{1} H\right)$. (The indices refer to the respective components.) Moreover, in system (0.1) $\varepsilon$ is a function that takes positive definite bounded matrix values, $\mu$ is a strictly positive bounded real valued function and $J$ and $K$ are force densities. In the case where there are no nontrivial solutions of problem (0.1), (0.2) with $J=0, K=0$, the approximation of the electric field $E$ is handled by Saranen in [17] for smooth and in [18] for polygonal domains of the plane. The approximation of the whole solution $(E \mid H)$ of problem $(0.1),(0.2)$ for polygonal $G \subset \boldsymbol{R}^{2}$ has been studied by Neittaanmäki and Saranen in [12].

In this paper we consider the finite element approximation of the eigenvalue problem arising from equation $(0.1),(0.2)$ in smooth domains. We denote

$$
M U:=i\left(\varepsilon^{-1} \operatorname{curl}_{*} H \mid-\mu^{-1} \operatorname{curl} E\right), U:=(E \mid H)
$$

and our problem can be written

$$
(M-\omega) U=0, \quad n \wedge U_{1 \mid \Gamma}=0 .
$$


It is well known ([8], [9], [10], [14]) that the resolvent of $M$, interpreted as an operator in a certain Hilbert space, is compact so that the spectrum of $M$ is purely discrete. We shall, in fact, first consider the approximation of eigenvalues and eigenvectors of the operator $\mathscr{A}:=M^{2}+1$ and reduce the situation back to the original problem (see Chapters 3 and 4).

Our method to prove error estimates for the approximation of eigenvalues is based on the use of the min-max-principle. This idea has been presented by Strang and Fix in [19] and has been developed later on to cover conforming and nonconforming schemes by many authors (see [1], [6], [7]). Our method follows partly the works of Kikuchi [7] and Ishihara [6] combined with some projection methods of Hilkert spaces presented in [10], [14] and [15]. In this form our method can be applied e.g. to the eigenvalue approximation of boundary value problems arising in [11]. For other general treatments of eigenvalue approximation we refer to [2], [3], [4], [13] and [20].

In the approximation of eigenvectors of Maxwell's equations with boundary condition of total reflection there arise some special difficulties. As the first aspect we mention that the electric field component $E$ of the eigensolutions is solenoidal (divergence free). For the approximation of eigensolutions we use piecewise linear continuous vector fields such that the first components satisfy the condition (0.2) at boundary nodes of the triangulation, but are solenoidal only asymptotically. In order to use regularity results we need to use smooth domains. Therefore the special boundary condition makes the nonconformal method necessary. Because of this, care should be taken especially in the evaluation of the lower bounds of eigenvalues. The reduction of the bilinear form problem (connected with $\mathscr{A}=M^{2}+1$ ) back to the original question of the eigenvalue problem of $M$ leads to difficulties, which are treated in Chapters 4.2 and 4.3.

Let us remark that the abstract methods of Chapters 3 and 4 are, with the exception of Lemma 3.1, independent of the dimension of the space. The reason why we restrict our discussions to the two dimensional case is that up to now the finite element approximations for the resolvent of $M$ are known only in the two dimensional case.

After submitting this paper the article [21] appeared. There finite element approximation of vector fields given by curl and divergence was considered in the three dimensional case.

\section{The eigenvalue problem}

1.1. In order to use variational methods we formulate the eigenvalue problem in a weak sense. The familiar Sobolev spaces $H^{k}(G)\left(k \in N, H^{0}(G)=L^{2}(G)\right)$ and $H_{0}^{1}(G)$ are needed for the precise formulation. The inner product of $H^{k}(G)$ is denoted by $(\cdot, \cdot)_{H^{k}(G)}$ with corresponding norm $\|\cdot\|_{H^{k}(G)}, k=0,1,2$. The subscript 
will be omitted in the case $k=0$. We will write $H^{k}(G),(\cdot, \cdot)_{H^{k}(G)}$ and $\|\cdot\|_{H^{k}(G)}$ also for the product spaces $H^{k}(G) \times \ldots \times H^{k}(G)$ without indicating the number of components which will always be clear from the context. Furthermore, we introduce in the same manner as in [9] the following special conventions

and

$$
\begin{aligned}
D_{\varepsilon} & :=\left\{V \in H^{0}(G) \mid \operatorname{div} \varepsilon V \in H^{0}(G)\right\}, \\
D_{0, \varepsilon} & :=\left\{V \in D_{\varepsilon} \mid \operatorname{div} \varepsilon V=0\right\},
\end{aligned}
$$

$$
\begin{aligned}
R & :=\left\{V \in H^{0}(G) \mid \operatorname{curl} V \in H^{0}(G)\right\}, \\
R_{*} & :=\left\{\varphi \in H^{0}(G) \mid \operatorname{curl}_{*} \varphi \in H^{0}(G)\right\}, \\
R^{0} & :=\left\{V \in R \mid\left(\operatorname{curl}_{*} \varphi, V\right)=(\varphi, \operatorname{curl} V) \text { for all } \varphi \in R_{*}\right\} .
\end{aligned}
$$

The derivates $\operatorname{div} \varepsilon V$, curl $V$ and $\operatorname{curl}_{*} \varphi$ appearing above are defined distributionally in an obvious way corresponding to the formulae $\operatorname{div} \varepsilon V=\partial_{i}\left(\varepsilon_{i j} V_{j}\right)$, curl $V=$ $\partial_{1} V_{2}-\partial_{2} V_{1}$ and $\operatorname{curl}_{*} \varphi=\left(\partial_{2} \varphi \mid-\partial_{1} \varphi\right)$, where $\partial_{i}:=\partial / \partial x_{i}, i=1,2$, and $(\cdot \mid \cdot)$ denotes the ordered pair.

With this terminology, we will need to make use of the Hilbert space

with the inner product

$$
\mathscr{H}_{*, s}:=\left(R \cap D_{\varepsilon} \times R_{*} \mid(\cdot, \cdot)_{*, s}\right)
$$

where

$$
(U, V)_{*, s}:=(U, V)_{s}+(M U, M V)
$$

and

$$
(U, V):=\left(U_{1}, \varepsilon V_{1}\right)+\left(U_{2}, \mu V_{2}\right)
$$

$$
(U, V)_{s}:=(U, V)+s\left(\operatorname{div} \varepsilon U_{1}, \operatorname{div} \varepsilon V_{1}\right)
$$

with a real parameter $s \geqq 0$. Moreover, we denote

and

$$
\begin{aligned}
& \mathscr{H}:=\left(H^{0}(G) \times H^{0}(G) \mid(\cdot, \cdot)\right) \\
& \mathscr{H}_{s}:=\left(D_{\varepsilon} \times H^{0}(G) \mid(\cdot, \cdot)_{s}\right)
\end{aligned}
$$

$$
\mathscr{H}^{0}:=\left\{V \in \mathscr{H}_{s} \mid V_{1} \in D_{0, \varepsilon}\right\} .
$$

1.2. Using the formal differential operator $M$, we now define the (symmetric) Maxwell operator

$$
\mathscr{M}: D(\mathscr{M}) \subset \mathscr{H}^{0} \rightarrow \mathscr{H}^{0}, \quad U \rightarrow M U,
$$

where $D(\mathscr{M}):=\left(R^{0} \times R_{*}\right) \cap \mathscr{H}^{0}$.

We denote by $N(\mathscr{M})$ the kernel of $\mathscr{M}$ and by $W(\mathscr{M})$ the range of $\mathscr{M}$. According to [8], [10] and [14] one has the results

(i) $\mathscr{M}$ is selfadjoint in $\mathscr{H}^{0}$ so that the spectrum $\sigma(\mathscr{M})$ of $\mathscr{M}$ is real.

(ii) The resolvent of $\mathscr{M}$ is compact, which implies the spectrum of $\mathscr{M}$ consists of isolated points. 
(iii) Let $P_{\omega}$ be the projection of $\mathscr{H}^{0}$ onto the space of eigenfunctions of $\mathscr{M}$ corresponding to $\omega$. Then

$$
P_{\omega} \perp P_{\lambda} \quad \omega \neq \lambda
$$

in the sense of the scalar product $(\cdot, \cdot)$.

We shall prove an important

Theorem 1.1. If $\omega^{2} \in \sigma\left(\mathscr{M}^{2}\right)$, then $\omega \in \sigma(\mathscr{M})$ and $-\omega \in \sigma(\mathscr{M})$. Let $Q_{\lambda}$ be the projection on the space of eigenfunctions of $\mathscr{A}:=\mathscr{M}^{2}+1$ corresponding to $\lambda:=\omega^{2}+1$, $\omega \geqq 0$. Then

and

$$
Q_{\lambda}=P_{\omega}+P_{-\omega} \text { for } \omega \neq 0
$$

$$
Q_{1}=P_{0} .
$$

Proof. A. If $\omega^{2} \in \sigma\left(\mathscr{M}^{2}\right)$, then $\omega \in \sigma(\mathscr{M})$ or $-\omega \in \sigma(\mathscr{M})$. If the ordered pair $\left(U_{1} \mid U_{2}\right)$ belongs to $W\left(P_{\omega}\right)=N(\mathscr{M}-\omega)$, it is easily seen that $\left(U_{1} \mid-U_{2}\right) \in W\left(P_{-\omega}\right)=$ $N(\mathscr{M}+\omega)$. Thus $\omega \in \sigma(\mathscr{M})$ and $-\omega \in \sigma(\mathscr{M})$.

B. In order to prove the second assertion of Theorem 1.1 let $U$ belong to $N(\mathscr{A}-\lambda) \equiv N\left(\mathscr{M}^{2}-\omega^{2}\right)$. Then

$$
F=(\mathscr{M}+\omega) U \in N(\mathscr{M}-\omega) .
$$

Because $\mathscr{M}$ is selfadjoint,

$$
\mathscr{H}^{0}=N(\mathscr{M}+\omega) \oplus W(\mathscr{M}+\omega),
$$

and it holds for all $U \in \mathscr{H}^{0}$ that

$$
U=U_{1}+U_{2} \in N(\mathscr{M}+\omega) \oplus W(\mathscr{M}+\omega),
$$

where the indices refer to the projection on the respective subspace. Thus, by (1.2),

$$
F=(\mathscr{M}+\omega) U_{2} .
$$

On the other hand, it holds by (1.2) that

$$
\begin{aligned}
(\mathscr{M}+\omega) F & =(\mathscr{M}-\omega) F+2 \omega F \\
& =2 \omega F .
\end{aligned}
$$

According to (1.4) and (1.5) we have for $\omega \neq 0, U_{2}-(2 \omega)^{-1} F \in N(\mathscr{M}+\omega)$. But (1.3) and (1.4) imply $U_{2}-(2 \omega)^{-1} F \in W(\mathscr{M}+\omega)$. Thus

$$
U_{2}=(2 \omega)^{-1} F \in N(\mathscr{M}-\omega),
$$

and therefore $Q_{\lambda}=P_{\omega}+P_{-\omega}$, according to (1.3).

The case $\omega=0$ is trivial: If $\mathscr{M}^{2} U=0$, then $\mathscr{M} U \in N(\mathscr{M}) \cap W(\mathscr{M})=\{0\}$. Thus $Q_{1}=P_{0}$.

According to Theorem 1.1 it is obviously sufficient to study the spectrum of $\mathscr{M}^{2}$ in order to obtain exact information as to the spectrum of $\mathscr{M}$. Without loss of 
generality we can move the spectrum to the right so that we can, in fact, study the properties of the spectrum of

$$
\mathscr{A}:=\mathscr{M}^{2}+1 \text {. }
$$

In what follows we are interested in the spectral points of $\mathscr{A}$ which lie between 1 and $\lambda_{0}$, where $\lambda_{0}$ is any fixed constant larger than 1 .

We shall give a variational formulation to this eigenvalue problem, which is based on the following theorem:

Theorem 1.2. Let

$$
\mathscr{K}=\min \left\{\|\nabla \varphi\|^{2} /\|\varphi\|^{2} \mid \varphi \in H_{0}^{1}(\Omega)\right\},
$$

$s=2 \mathscr{K}^{-1}\left(\lambda_{0}-1\right)$, and let $\lambda \in\left[1, \lambda_{0}\right]$. Then $U \in N(\mathscr{A}-\lambda)$ if and only if the equation

holds.

$$
(\Phi, U)_{*, s}=\lambda(\Phi, U) \text { for all } \Phi \in D(\mathscr{M})
$$

Proof. We note that, if $U \in N(\mathscr{A}-\lambda)$, $\operatorname{div} \varepsilon U_{1}=0$ and $\varepsilon^{-1} \operatorname{curl}_{*} U_{2} \in R^{0}$. By partial integration we find that $U$ satisfies (1.6).

Conversely, the argument follows the same lines as in the proof of Theorem 1.3 in [12] (see also [10]).

Let us remark that we can indeed determine the "elliptization parameter" $s$, since $\mathscr{K}$ is the first eigenvalue of the (negative) Laplacian and its evaluation is well known in many respects (see [5], [19]).

We are now in a position to define our problem in variational form:

Problem (EP). Find the eigenvalues $\lambda \in\left[1, \lambda_{0}\right]$ and the eigenfunctions $U$ such that

$$
(\Phi, U)_{*, s}=\lambda(\Phi, U) \text { for all } \Phi \in D(\mathscr{M}) .
$$

The Problem (EP) admits a finite sequence of positive eigenvalues $\left\{\lambda_{i}\right\}_{i}$ each of finite, even multiplicity $\left\{v_{j}\right\}_{j}$ (we agree that eigenvalues are ordered and repeated according to their multiplicity) and a corresponding sequence of eigenfunctions $\left\{U_{i}\right\}_{i}$ with the normalization condition

$$
\left(U_{i}, U_{j}\right)=\delta_{i j}
$$

Let $E(\lambda)$ be the spectral family of $\mathscr{A}$ and let

$$
Q_{\lambda}:=E(\lambda+)-E(\lambda-) \text {. }
$$

We define on $\mathscr{H}_{*, s}-\{0\}$ the Rayleigh quotient

$$
\mathscr{R}(\Phi)=\frac{\|\Phi\|_{*, s}^{2}}{\|\Phi\|^{2}}
$$

The eigenvalues of the Problem (EP) can be characterized by the Rayleigh principle: The stationary points of $\mathscr{R}$ are precisely the eigenfunction of the Problem (EP) and 
the values of $\mathscr{R}$ at such points are the corresponding eigenvalues:

$$
\lambda_{i}=\min \left\{\mathscr{R}(\Phi) \mid \Phi \in W\left(E\left(\lambda_{i-1}+\right)\right)^{\perp}, \Phi \neq 0\right\} .
$$

Moreover, $\mathscr{R}(\Phi)=\lambda$ for every $\Phi \in W\left(Q_{\lambda}\right)$ and $\operatorname{dim} W\left(Q_{\lambda_{i}}\right)=v_{j}, 2 \leqq v_{j}<\infty$ for all $\lambda_{i}=\lambda_{k}$ with $k=\sum_{\ell=1}^{j-1} v_{\ell}+1$.

By the regularity results in [10], Theorem 3.2, and by (1.7), it holds for every eigensolution $U_{\lambda}$ of (1.6) that $U_{\lambda} \in H^{2}(G)$ and that

$$
\left\|U_{\lambda}\right\|_{H^{2}(G)} \leqq c \lambda .
$$

Note that, as above, we adapt the usual convention that $c, c_{1}, c_{2}, \ldots$ always denote positive generic constants, which may vary from context to context.

\section{Finite element approximation of problem (EP)}

To approximate the eigenfunctions and eigenvectors of (1.6) we introduce a family of finite dimensional subspaces $\mathscr{H}^{h} \subset \mathscr{H}_{*, s}$ depending on a discretization parameter $0<h<1$ going to zero. The idea is to solve (1.6) on these subspaces.

For a precise formulation, let $\mathscr{T}_{h}$ be a family of regular triangulations of $G$ in the usual sense ([10])

$$
\bar{G}=\cup\left\{\bar{T}_{h} \mid T_{h} \in \mathscr{T}_{h}\right\} .
$$

Let $K_{h}$ be the set of all nodes of $\mathscr{T}_{h}$ lying on the boundary. Denoting $C(\bar{G})$ := $\{\varphi: \bar{G} \rightarrow C \mid \varphi$ continuous $\}$ we define, as in [10], and

$$
S_{1}^{h}:=\left\{\Phi=\left(\Phi_{1} \mid \Phi_{2}\right) \in C(\bar{G}) \times C(\bar{G})\left|\Phi_{i}\right|_{T_{h}} \text { linear, }(n \wedge \Phi)(x)=0, x \in K_{h}\right\},
$$

$$
S_{2}^{h}:=\left\{\varphi \in C(\bar{G})|\varphi|_{T_{h}} \text { linear }\right\} .
$$

We define $\mathscr{H}^{h}:=S_{1}^{h} \times S_{2}^{h}$ as a subspace of $\mathscr{H}_{*, s}$.

The discrete analogue of Problem (EP) reads:

Problem $(\mathrm{EP})^{h}$. Find the eigenvalues $\lambda^{h} \in[1, \infty)$ and the eigenfunctions $U^{h} \in \mathscr{H}^{h}$ such that

$$
\left(\Phi, U^{h}\right)_{*, s}=\lambda^{h}\left(\Phi, U^{h}\right) \text { for all } \Phi \in \mathscr{H}^{h} .
$$

Since $\mathscr{H}^{h} \pitchfork D(\mathscr{M}),(2.1)$ is a nonconforming finite element model for solving Problem (EP).

In order to write (2.1) as an equivalent operator equation, we recall some definitions from [10]. Let $P_{*}$ be the orthogonal projection on $R^{0} \cap D_{\varepsilon} \times R_{*}$ defined through the decomposition

$$
\mathscr{H}_{*, s}=R^{0} \cap D_{\varepsilon} \times R_{*} \oplus N\left(\operatorname{div} \mu^{-1} \operatorname{curl}_{*}+\varepsilon\right) \times\{0\} .
$$


We introduce the operators

by equations

$$
\begin{aligned}
S: \mathscr{H} & \rightarrow P_{*} \mathscr{H}_{*, s}, & T: \mathscr{H} & \rightarrow \mathscr{H}_{*, s} \\
\Phi & \rightarrow \psi^{(1)} & \Phi & \rightarrow \psi^{(2)}
\end{aligned}
$$

$$
\left(\eta, \psi^{(1)}\right)_{*, s}=(\eta, \Phi) \text { for all } \eta \in P_{*} \mathscr{H}_{*, s}
$$

and, respectively,

$$
\left(\eta, \psi^{(2)}\right)_{*, s}=\left(\left(1-P_{*}\right) \eta, \Phi\right) \text { for all } \Phi \in \mathscr{H}_{*, s} .
$$

Let $P^{h}$ be the orthogonal projection of $\mathscr{H}_{*, s}$ on $\mathscr{H}^{h}$. It is easily seen that equation (2.1) is equivalent to

$$
\left(I-\lambda^{h} P^{h}(S+T)\right) U^{h}=0 .
$$

According to [10], Theorem 2.6, the pseudoinverse

$$
\mathscr{A}_{h}:=\left(P^{h}(S+T)\right)^{-1}: \mathscr{H}^{h} \rightarrow \mathscr{H}^{h}
$$

exists and Problem (EP) ${ }^{h}$ corresponds to the problem

$$
\left(\mathscr{A}_{h}-\lambda^{h}\right) U^{h}=0 .
$$

We note that with respect to $\mathscr{H} \mathscr{A}_{h}$ is a symmetric, finite dimensional operator and let $E^{h}(\lambda)$ be its spectral family.

Following the same lines as in [19] it can be proved:

Theorem 2.1. Problem (EP) ${ }^{h}$ admits $N_{h}:=\operatorname{dim} \mathscr{H}^{h}$ real, possibly repeated, eigenvalues $\left\{\lambda_{j}\right\}_{j=1}^{N_{h}}$ (arranged in increasing order $1 \leqq \lambda_{1}^{h} \leqq \lambda_{2}^{h} \leqq \ldots \leqq \lambda_{N^{h}}^{h}<\infty$ ) and corresponding eigenfunctions $\left\{U_{j}^{h}\right\}_{j=1}^{N_{h}}$ with the normalization condition

$$
\left(U_{i}^{h}, U_{j}^{h}\right)=\delta_{i j}
$$

Moreover, the following characterizations hold:

$$
\lambda_{i}^{h}=\min \left\{\mathscr{R}(\Phi) \mid \Phi \in W\left(E^{h}\left(\lambda_{i-1}^{h}+\right)\right)^{\perp}, \Phi \neq 0\right\},
$$

$\mathscr{R}\left(U_{j}^{h}\right)=\lambda_{j}^{h}$ and (min-max-principle)

$$
\lambda_{i}^{h}=\min _{\mathscr{H}_{i}^{h} \cong \mathscr{H}^{h}} \max _{\Phi \in \mathscr{H}_{i}^{h}} \mathscr{R}(\Phi),
$$

where $\mathscr{H}_{i}^{h}$ is an $i$-dimensional subspace of $\mathscr{H}^{h}$. Then

Let $\widetilde{Q}_{\mu^{h}}^{h}$ be the projection on the eigenspace of $\mathscr{A}_{h}$ relative to eigenvalue $\mu^{h}$.

and it holds that

$$
E^{h}(\lambda)=\sum_{\mu^{h} \leqq \lambda} \widetilde{Q}_{\mu^{h}}^{h}
$$

$$
\left(\Phi, \widetilde{Q}_{\mu^{h}}^{h} U\right)_{*, s}=\mu^{h}\left(\Phi, \tilde{Q}_{\mu^{h}}^{h} U\right) \quad \text { for all } \Phi \in \mathscr{H}^{h}
$$




\section{Error bounds for eigenvalue approximation}

Let $m$ be the number of the eigenvalues of $\mathscr{A}$ lying in $\left[1, \lambda_{0}\right]$. In this section we shall give error bounds to approximation of the eigenvalues $\lambda_{i}(i=1, \ldots, m)$ by the eigenvalues $\lambda_{i}^{h}$ in $\left[1, \lambda_{0}+1\right]$.

At first we prove as a corollary to the abstract approximation results given in Section 3 of [10].

Lemma 3.1. Let

$$
A_{\lambda}^{h}:=P^{h}+P^{h} T \mathscr{A}: W(E(\lambda+)) \rightarrow W\left(A_{\lambda}^{h}\right) \subset \mathscr{H}^{h} .
$$

Then the asymptotic estimates

$$
\begin{gathered}
\left\|\left(I-A_{\lambda}^{h}\right) U\right\| \leqq c h^{2}\|U\|_{H^{2}(G)} \\
\left\|\left(I-A_{\lambda}^{h}\right) U\right\|_{*, s} \leqq c h\|U\|_{H^{2}(G)}
\end{gathered}
$$

hold for $U \in W(E(\lambda+))$. Furthermore,

for sufficiently small $h$.

$$
\operatorname{dim}\left(W\left(A_{h}^{\lambda}\right)\right)=\operatorname{dim}(W(E(\lambda+)))
$$

Proof. A. By [10], Section 3,

$$
\left\|\left(I-P^{h}\right) V\right\|+h\left\|\left(I-P^{h}\right) V\right\|_{*, s} \leqq c h^{2}\|V\|_{H^{2}(G)}
$$

holds for all $V \in P_{*} \mathscr{H}_{*, s} \cap H_{2}(G)$ and

$$
\left\|P^{h} T Y\right\|+h\left\|P^{h} T Y\right\|_{*, s} \leqq c h^{2}\|Y\|
$$

for all $Y \in \mathscr{H}$.

Using the regularity (1.10) we have $U \in H^{2}(G)$ for every $U \in W(E(\lambda+))$. Thus the estimates (3.1) and (3.2) follow from (3.3) and (3.4).

B. To have the last assertion, taking into account that $\operatorname{dim} A_{\lambda}^{h}(W(E(\lambda+)))=$ $\operatorname{dim} W(E(\lambda+))-\operatorname{dim} N\left(A_{\lambda}^{h}\right)$, we only have to prove that $A_{\lambda}^{h}: W(E(\lambda+)) \rightarrow W\left(A_{\lambda}^{h}\right)$ is injective.

If $A_{\lambda}^{h} U=0$, we have $U=\left(I-P^{h}\right) U-P^{h} T \mathscr{A}$. Estimates (3.3) and (3.4) yield

$$
\|U\| \leqq c h^{2}\|U\|_{H^{2}(G)} .
$$

By the regularity argument we obtain $\|U\| \leqq c h^{2}\|U\|$. Therefore $U=0$ if $h$ is small enough.

Using the min-max-principle and Lemma 3.1 we can prove the error estimate for the approximations of eigenvalues:

Theorem 3.2. Let $\lambda_{i}^{h}$ be the approximate eigenvalue of $\lambda_{i}(i=1, \ldots, m)$. Then, for sufficiently small $h$, it holds that

$$
\left|\lambda_{i}-\lambda_{i}^{h}\right| \leqq c \lambda_{0}^{2} h^{2} .
$$


Proof. A. We shall first prove

$$
\lambda_{i}^{h} \leqq \lambda_{i}+c \lambda_{0}^{2} h^{2} .
$$

Using the definitions of $S$ and $T$, given in (2.2) and (2.3), respectively, and the orthogonality $Q_{\lambda} \perp Q_{\mu}, \lambda \neq \mu$, we obtain for $F_{i} \in W\left(E\left(\lambda_{i}+\right)\right), F_{i}=\Sigma_{1 \leqq \lambda \leqq \lambda_{i}} Q_{\lambda} F_{i}$,

$$
\begin{aligned}
& \left\|A_{\lambda_{i}}^{h} F_{i}\right\|_{*, s}^{2}=\left(A_{\lambda_{i}}^{h} F_{i},(I+T \mathscr{A}) F_{i}\right)_{*, s} \\
= & \left(A_{\lambda_{i}}^{h} F_{i},(S+T) \mathscr{A} F_{i}\right)_{*, s}=\left(A_{\lambda_{i}}^{h} F_{i}, \mathscr{A} F_{i}\right) \\
\leqq & \left\|A_{\lambda_{i}}^{h} F_{i}\right\|\left\|\mathscr{A} F_{i}\right\| .
\end{aligned}
$$

Inequality (3.1) implies $A_{\lambda_{i}}^{h} F_{i} \neq 0$ and $\left\|A_{\lambda_{i}}^{h} F_{i}\right\|^{-1}>2^{-1}$, for sufficiently small $h$. By (3.7), (3.1), and by regularity,

holds. Heilce

$$
\begin{aligned}
\left\|A_{\lambda_{i}}^{h} F_{i}\right\|_{*, s}^{2} & \leqq \lambda_{i}\left\|A_{\lambda_{i}}^{h} F_{i}\right\|\left(\left\|A_{\lambda_{i}}^{h} F_{i}\right\|+\left\|\left(I-A_{\lambda}^{h}\right) F_{i}\right\|\right) \\
& \leqq \lambda_{i}\left\|A_{\lambda_{i}}^{h} F_{i}\right\|^{2}\left(1+c \lambda_{i} h^{2}\right)\left\|F_{i}\right\|
\end{aligned}
$$

$$
\mathscr{R}\left(A_{\lambda_{i}}^{h} F_{i}\right) \leqq \lambda_{i}+c \lambda_{i}^{2} h^{2} \leqq \lambda_{i}+c \lambda_{0}^{2} h^{2} .
$$

By Lemma $3.1 \operatorname{dim} W\left(A_{\lambda_{i}}^{h}\right)=\sigma_{k}, \sigma_{k}:=\sum_{j=1}^{k} v_{j}, \sum_{\ell=1}^{k-1} v_{\ell}+1 \leqq i \leqq \sum_{\ell=1}^{k} v_{\ell}$ and using the min-max-principle, we get $\lambda_{i}^{h} \leqq \lambda_{\sigma_{k}}^{h} \leqq \lambda_{i}+c \lambda_{0}^{2} h^{2}$.

B. By (3.6) we only have to prove

$$
\lambda_{i}-c \lambda_{i}^{2} h^{2} \leqq \lambda_{i}^{h}
$$

for sufficiently small $h$.

Let $U^{h} \in \mathscr{H}_{\sigma}^{h} \cap W\left(E\left(\lambda_{\sigma_{k-1}}+\right)\right)^{\perp}\left(H_{\sigma}^{h} \subset \mathscr{H}^{h}\right.$ with $\operatorname{dim}\left(\mathscr{H}_{c}^{h}\right)=\sigma=\sum_{\ell=1}^{k-1} v_{\ell}+1$; the intersection is indeed nonempty, since the condition $\left(U^{h}, U_{j}\right)=0, j=1, \ldots, \sigma_{k-1}$, contains $\sigma_{k-1}$ restrictions (unknowns), $\sigma_{k-1}$ and $i$ as above). Since for all $F_{i} \in W\left(E\left(\lambda_{i-1}+\right)\right)$

$$
\left(S U^{h}, F_{i}\right)_{*, s}=\sum_{1 \leqq \lambda \leqq \lambda_{\sigma_{k-1}}}\left(S U^{h}, Q_{\lambda} F_{i}\right)_{*, s}=\sum_{1 \leqq \lambda \leqq \lambda_{\sigma_{k-1}}} \lambda^{-1}\left(U^{h}, Q_{\lambda} F_{i}\right)=0,
$$

we have $S U^{h} \in W\left(E\left(\lambda_{\sigma_{k-1}}\right)\right)^{\perp}$. By (2.2) and Rayleigh's principle (1.9) we obtain

which implies

$$
\begin{aligned}
\lambda_{i}^{2}\left\|S U^{h}\right\|^{4} & \leqq\left\|S U^{h}\right\|_{*, s}^{4}=\left(S U^{h}, S U^{h}\right)_{*, s}^{2} \\
& \leqq\left\|U^{h}\right\|^{2}\left\|S U^{h}\right\|^{2} \leqq\left\|U^{h}\right\|^{2} \lambda_{i}^{-1}\left\|S U^{h}\right\|_{*, s}^{2},
\end{aligned}
$$

$$
\left\|S U^{h}\right\|^{2} \leqq \lambda_{i}^{-2}\left\|U^{h}\right\|^{2} \text { and }\left\|S U^{h}\right\|_{*, s}^{2} \leqq \lambda_{i}^{-1}\left\|U^{h}\right\|^{2} .
$$

Since, according to [10], Section 3 ,

$$
\left\|S U^{h}-P^{h}(S+T) U^{h}\right\| \leqq c h^{2}\left\|U^{h}\right\|,
$$


we conclude by the formulae (2.2), (2.3) and (3.9)

If $c \lambda_{i} h^{2} \leqq 1$, this implies

$$
\begin{aligned}
\left\|U^{h}\right\|^{4} & =\left|\left(U^{h}, P^{h}(S+T) U^{h}\right)_{*, s}\right|^{2} \\
& \leqq\left\|U^{h}\right\|_{*, s}^{2}\left\|P^{h}(S+T) U^{h}\right\|_{*, s}^{2} \\
& =\left\|U^{h}\right\|_{*, s}^{2}\left|\left(P^{h}(S+T) U^{h}, U^{h}\right)\right| \\
& \leqq\left\|U^{h}\right\|_{*, s}^{2}\left\|U^{h}\right\| \lambda_{i}^{-1}\left(1+c \lambda_{i} h^{2}\right) .
\end{aligned}
$$

$$
\mathscr{R}\left(U^{h}\right) \geqq \lambda_{i}\left(1+c \lambda_{i} h^{2}\right)^{-1} \geqq \lambda_{i}-c \lambda_{i}^{2} h^{2} .
$$

Using the min-max-principle we obtain (3.8), since $\lambda_{\sigma}^{h} \leqq \lambda_{i}^{h}$. Estimate (3.5) follows if we combine (3.6) and (3.8).

We now want to obtain an approximation for the corresponding eigenvalue $\omega$ of operator $\mathscr{M}$. We recall that

Let

$$
\omega= \pm \sqrt{\lambda-1}
$$

$$
\omega^{h}:= \pm \sqrt{\lambda^{h}-1}
$$

We conclude by Theorem 3.2 for $\omega \neq 0$ and sufficiently small $h>0$

Thus we have

$$
\begin{gathered}
\left|\omega-\omega^{h}\right| \leqq c\left|\omega^{2}-\left(\omega^{h}\right)^{2}\right| \\
=c\left|\lambda-\lambda^{h}\right| \leqq c \lambda_{0} h^{2} .
\end{gathered}
$$

Theorem 3.3. Let $\omega$ be an eigenvalue of $\mathscr{M},|\omega|<\sqrt{\lambda_{0}-1}$, and let $\omega^{h}$ be its finite element approximation defined through equations (2.1) and (3.10). Then

$$
\left|\omega-\omega^{h}\right|=\mathcal{O}\left(h^{2}\right), \quad \text { for } \quad h \rightarrow 0 .
$$

\section{Error bounds for eigenfunction approximation}

4.1. Let $\lambda^{\prime}\left(\lambda^{\prime \prime}\right)$ be the greatest (smallest) eigenvalue with $\lambda^{\prime}<\lambda$ (with $\lambda^{\prime \prime}>\lambda$, respectively). According to Theorem 3.2 it holds for all eigenvalues $\lambda$ of Problem (EP) with $1 \leqq \lambda \leqq \lambda_{0}$ and for the corresponding $\lambda^{h}$ (i.e. the eigenvalue with the same index) of Problem (EP) $)^{h}$ with $1 \leqq \lambda^{h} \leqq \lambda_{0}+1$ that

$$
\lambda^{\prime}<\lambda-c \lambda_{0}^{2} h^{2} \leqq \lambda^{h} \leqq \lambda+c \lambda_{0}^{2} h^{2}<\lambda^{\prime \prime},
$$

for sufficiently small $h$.

We abbreviate

and define

$$
\lambda(h):=\lambda+c \lambda_{0}^{2} h|h|
$$

$$
Q_{\lambda}^{h}:=E^{h}(\lambda(h))-E^{h}(\lambda(-h)) .
$$


By (4.1) it holds that

$$
Q_{\lambda}^{h}=\sum_{\lambda(-h) \leqq \mu \leqq \lambda(h)} \widetilde{Q}_{\mu}^{h}
$$

Using Lemma 3.1 as an essential tool we prove

Theorem 4.1. The asymptotic error estimates

$$
\begin{gathered}
\left\|\left(I-Q_{\lambda}^{h}\right) Q_{\lambda} U\right\| \leqq c \lambda_{0}^{2} h^{2}\|U\|, \\
\left\|\left(I-Q_{\lambda}^{h}\right) Q_{\lambda} U\right\|_{*, s} \leqq c \lambda_{0}^{3 / 2} h\|U\|
\end{gathered}
$$

hold for all eigenvalues $\lambda, 1 \leqq \lambda \leqq \lambda_{0}$, of operator $\mathscr{A}$.

Proof. A. Let $\lambda^{\prime}, \lambda$ and $\lambda^{\prime \prime}$ be three eigenvalues such that $1 \leqq \lambda^{\prime}<\lambda<\lambda^{\prime \prime} \leqq \lambda_{0}$, $1=\lambda<\lambda^{\prime \prime}$ or that $\lambda^{\prime}<\lambda=\lambda_{0}$, satisfying (4.1). Using relation

$$
I=E^{h}\left(\lambda^{\prime}(h)\right)+Q_{\lambda}^{h}+\left(I-E^{h}\left(\lambda^{\prime \prime}(-h)\right)\right),
$$

Lemma 3.1 and the regularity estimate (1.10) we conclude

$$
\begin{aligned}
&\left\|\left(I-Q_{\lambda}^{h}\right) Q_{\lambda} U\right\| \leqq\left\|\left(I-A_{\lambda}^{h}\right) Q_{\lambda} U\right\|+\left\|\left(A_{\lambda}^{h}-Q_{\lambda}^{h}\right) Q_{\lambda} U\right\| \\
& \leqq c \lambda h^{2}\|U\|+\left\|E^{h}\left(\lambda^{\prime}(h)\right) A_{\lambda}^{h} Q_{\lambda} U\right\| \\
&+\left\|\left(I-E^{h}\left(\lambda^{\prime \prime}(h)\right)\right) A_{\lambda}^{h} Q_{\lambda} U\right\| \\
&=: c \lambda h^{2}\|U\|+(1)+(2) .
\end{aligned}
$$

We will prove that

$$
\text { (1) } \leqq \frac{2 \lambda}{\lambda-\lambda^{\prime}}\left\|\left(I-A_{\lambda}^{h}\right) Q_{\lambda} U\right\|
$$

and that

$$
\text { (2) } \leqq \frac{2 \lambda}{\lambda^{\prime \prime}-\lambda}\left\|\left(I-A_{\lambda}^{h}\right) Q_{\lambda} U\right\|
$$

If we combine (4.6), (4.7) and (4.8) with Lemma 3.1, the inequality (4.3) follows.

B. Let us first prove (4.7). By (2.9)

$$
\begin{gathered}
\left(\left(I-E^{h}\left(\lambda^{\prime}(h)\right)\right) A_{\lambda}^{h} Q_{\lambda} U, E^{h}\left(\lambda^{\prime}(h)\right) A_{\lambda}^{h} Q_{\lambda} U\right)_{*, s} \\
=\sum_{1 \leqq \mu \leqq \lambda^{\prime}(h)}\left(A_{\lambda}^{h} Q_{\lambda} U, \widetilde{Q}_{\mu}^{h} U\right) \mu\left(\left(I-E^{h}\left(\lambda^{\prime}(h)\right)\right) A_{\lambda}^{h} Q_{\lambda} U, \widetilde{Q}_{\mu}^{h} U\right)=0 .
\end{gathered}
$$

Using this with the identity $I=E^{h}\left(\lambda^{\prime}(h)\right)+\left(I-E^{h}\left(\lambda^{\prime}(h)\right)\right)$ we find

$$
\begin{gathered}
\left\|E^{h}\left(\lambda^{\prime}(h)\right) A_{\lambda}^{h} Q_{\lambda} U\right\|_{*, s}^{2} \\
=\left(E^{h}\left(\lambda^{\prime}(h)\right) A_{\lambda}^{h} Q_{\lambda} U, A_{\lambda}^{h} Q_{\lambda} U\right)_{*, s} \\
=\lambda\left(E^{h}\left(\lambda^{\prime}(h)\right) A_{\lambda}^{h} Q_{\lambda} U, Q_{\lambda} U\right) .
\end{gathered}
$$

Moreover,

$$
\left(E^{h}\left(\lambda^{\prime}(h)\right) A_{\lambda}^{h} Q_{\lambda} U, A_{\lambda}^{h} Q_{\lambda} U\right)=\left\|E^{h}\left(\lambda^{\prime}(h)\right) A_{\lambda}^{h} Q_{\lambda} U\right\|^{2} .
$$



$\lambda \neq \mu$,

On the other hand, we obtain by (2.9) and by the orthogonality $\widetilde{Q}_{\lambda}^{h} \perp \widetilde{Q}_{\mu}^{h}$,

$$
\begin{gathered}
\left\|E^{h}\left(\lambda^{\prime}(h)\right) A_{\lambda}^{h} Q_{\lambda} U\right\|_{*, s}^{2} \\
=\sum_{1 \leqq \mu \leqq \lambda^{\prime}(h)} \mu\left(A_{\lambda}^{h} Q_{\lambda} U, \tilde{Q}_{\mu}^{h} U\right)^{2}\left(\widetilde{Q}_{\mu}^{h} U, \tilde{Q}_{\mu}^{h} U\right) \\
\equiv \lambda^{\prime}(h)\left\|E^{h}\left(\lambda^{\prime}(h)\right) A_{\lambda}^{h} Q_{\lambda} U\right\|^{2} .
\end{gathered}
$$

A combination of (4.10), (4.11) and (4.12) yields

$$
\begin{gathered}
\left(\lambda-\lambda^{\prime}(h)\right)\left\|E^{h}\left(\lambda^{\prime}(h)\right) A_{\lambda}^{h} Q_{\lambda} U\right\|^{2} \\
\leqq \\
\lambda\left(E^{h}(\lambda(h)) A_{\lambda}^{h} Q_{\lambda} U, A_{\lambda}^{h} Q_{\lambda} U-Q_{\lambda} U\right),
\end{gathered}
$$

which implies (4.7) for sufficiently small $h$.

C. In order to prove (4.8) we first find by arguments similar to (4.10) that

$$
\begin{gathered}
\\
\|\left(I-E^{h}\left(\lambda^{\prime \prime}(-h)\right) A_{\lambda}^{h} Q_{\lambda} U \|_{*, s}^{2}\right. \\
=\lambda\left(\left(I-E^{h}\left(\lambda^{\prime \prime}(-h)\right)\right) A_{\lambda}^{h} Q_{\lambda} U, Q_{\lambda} U\right) .
\end{gathered}
$$

Since $I-E^{h}\left(\lambda^{\prime \prime}(-h)\right)=I-E^{h}(\lambda(h))$, we have

and so by (2.6)

$$
\left(I-E^{h}\left(\lambda^{\prime \prime}(-h)\right)\right) A_{\lambda}^{h} Q_{\lambda} U \in W\left(E^{h}(\lambda(h))\right) \perp,
$$

$$
\begin{aligned}
& \tilde{\lambda}^{h}\left\|\left(I-E^{h}\left(\lambda^{\prime \prime}(-h)\right)\right) A_{\lambda}^{h} Q_{\lambda} U\right\|^{2} \\
\leqq & \left\|\left(I-E^{h}\left(\lambda^{\prime \prime}(-h)\right)\right) A_{\lambda}^{h} Q_{\lambda} U\right\|_{*, s}^{2},
\end{aligned}
$$

where $\tilde{\lambda}^{h}$ is the smallest eigenvalue for which $\tilde{\lambda}^{h} \geqq \lambda(h)$ holds. Also $\lambda^{h} \geqq \lambda^{\prime \prime}(-h)$.

The orthogonality yields

$$
\begin{gathered}
\left\|\left(I-E^{h}\left(\lambda^{\prime \prime}(-h)\right)\right) A_{\lambda}^{h} Q_{\lambda} U\right\|^{2} \\
=\left(\left(I-E^{h}\left(\lambda^{\prime \prime}(-h)\right)\right) A_{\lambda}^{h} Q_{\lambda} U, A_{\lambda}^{h} Q_{\lambda} U\right) .
\end{gathered}
$$

Thus we obtain from (4.13) and (4.14)

$$
\begin{gathered}
\left(\tilde{\lambda}^{h}-\lambda\right)\left\|\left(I-E^{h}\left(\lambda^{\prime \prime}(-h)\right)\right) A_{\lambda}^{h} Q_{\lambda} U\right\|^{2} \\
\leqq \lambda\left(\left(I-E^{h}\left(\lambda^{\prime \prime}(-h)\right)\right) A_{\lambda}^{h} Q_{\lambda} U, Q_{\lambda} U-A^{h} Q_{\lambda} U\right),
\end{gathered}
$$

which implies (4.8) for sufficiently small $h$.

D. We now prove the second assertion of Theorem 4.1. Again, by Lemma 3.1 and by regularity it holds that

$$
\begin{gathered}
\left\|Q_{\lambda} U-Q_{\lambda}^{h} Q_{\lambda} U\right\|_{*, s} \\
\leqq c \lambda h\left\|Q_{\lambda} U\right\|+\left\|A_{\lambda}^{h} Q_{\lambda} U-Q_{\lambda}^{h} Q_{\lambda} U\right\|_{*, s} .
\end{gathered}
$$

Using Theorem 3.1, regularity and the fact that

$$
\begin{gathered}
\left\|Q_{\lambda}^{h} U\right\|_{*, s}^{2}-\left(A_{\lambda}^{h} Q_{\lambda} U, Q_{\lambda}^{h} Q_{\lambda} U\right) \\
=\sum_{\lambda(-h) \leqq \mu \geqq \lambda(h)} \mu\left(Q_{\lambda}^{h} Q_{\lambda} U-A_{\lambda}^{h} Q_{\lambda} U, \tilde{Q}_{\mu}^{h} Q_{\lambda} U\right),
\end{gathered}
$$


we conclude

$$
\begin{gathered}
\left\|A_{\lambda}^{h} Q_{\lambda} U-Q_{\lambda}^{h} Q_{\lambda} U\right\|_{*, s}^{2} \\
\leqq c_{1} \lambda^{2} h^{2}\left\|Q_{\lambda} U\right\|+c_{2} \lambda^{2} \lambda(h)\left\|Q_{\lambda} U\right\|^{2} .
\end{gathered}
$$

This and inequality (4.15) yield (4.4).

4.2. Using our result for $\mathscr{A}=\mathscr{M}^{2}+1$ we now want to obtain error estimates for the eigenfunctions of operator $\mathscr{M}$ corresponding to the eigenvalue $\omega$. We shall first reformulate Theorem 4.1 with the help of Theorem 1.1.

According to Theorem 1.1 it holds that $Q_{\lambda} P_{\omega} U=P_{\omega} U$ and $Q_{\lambda} P_{-\omega} U=P_{-\omega}$. Let

$$
Z_{\omega}^{h}:=Q_{\lambda}^{h} P_{\omega}, \omega \in \sigma(\mathscr{M}), \lambda=\omega^{2}+1 .
$$

By Theorem 4.1 we have that

$$
\left\|P_{\omega} U-Z_{\omega}^{h} U\right\|+\lambda_{0}^{1 / 2} h\left\|P_{\omega} U-Z_{\omega}^{h} U\right\|_{*, s} \leqq c \omega_{0}^{2} h^{2}\left\|P_{\omega} U\right\|
$$

holds for all $-\omega_{0} \leqq \omega \leqq \omega_{0}, \omega_{0}:=\sqrt{\lambda_{0}^{2}-1}$.

4.3. Theorem 4.2 gives an approximation result for the spaces of eigenfunctions of operator $\mathscr{M}$. But unfortunately $Z_{\omega}^{h}$ is not constructive, because in order to know $Z_{\omega}^{h}$ we in fact should know $P_{\omega}$. On the other hand, $W\left(Q_{\lambda}^{h}\right)$ is constructive. One way to overcome this difficulty is to decompose $W\left(Q_{\lambda}^{h}\right)$ in a suitable way. To do this we must find appropriate orthogonal projectors $P_{\omega}^{h}$ and $P_{-\omega}^{h}$ such that

$$
Q_{\lambda}^{h}=P_{\omega}^{h}+P_{-\omega}^{h} .
$$

In order to construct $P_{\omega}^{h}$ and $P_{-\omega}^{h}$ we first remark that, since

$$
\mathscr{M} P_{ \pm \omega} U= \pm \omega P_{ \pm \omega} U
$$

holds, we have

$$
\operatorname{Re}\left(P_{\omega} U, \mathscr{M} P_{\omega} U\right)>0 \text { and } \operatorname{Re}\left(P_{-\omega} U, \mathscr{M} P_{-\omega} U\right)<0 .
$$

Let

and let

$$
\hat{\mathscr{R}}(\Phi):=\frac{\operatorname{Re}(\Phi, \mathscr{M} \Phi)}{\|\Phi\|^{2}}
$$

$$
\mu_{i}^{h}:=\min \left\{\hat{\mathscr{R}}(\Phi) \mid \Phi \in W\left(Q_{\lambda}^{h}\right), \Phi \neq 0,\left(\Phi, \Phi_{j}^{h}\right)=0, j=0,1, \ldots, i-1\right\},
$$

$i=1, \ldots, v=\operatorname{dim}\left(W\left(Q_{\lambda}^{h}\right)\right)=\operatorname{dim}\left(W\left(Q_{\lambda}\right)\right)$. Here $\Phi_{i}^{h} \in W\left(Q_{\lambda}^{h}\right), i=1, \ldots, v$, are the stationary points of $\hat{\mathscr{R}}$ with $\left(\Phi_{i}^{h}, \Phi_{j}^{h}\right)=\delta_{i j}$.

We define for $\omega>0$

$$
P_{\omega}^{h} U=\sum_{i=1}^{\nu / 2}\left(U, \Phi_{i}^{h}\right) \Phi_{i}^{h}
$$


and

$$
P_{-\omega}^{h} U=\sum_{i=v / 2+1}^{v}\left(U, \Phi_{i}^{h}\right) \Phi_{i}^{h} .
$$

Obviously, it holds that $P_{\omega}^{h} \perp P_{-\omega}^{h}$. For $\omega=0$ we set $P_{0}^{h}=Z_{0}^{h}$.

It will be shown that projections $P_{\omega}^{h}, \omega \in \sigma(\mathscr{M})$, are "approximations" of $P_{\omega}$ in a certain sense (similar to the connection between $Q_{\lambda}^{h}$ and $Q_{\lambda}$ ).

We shall first give asymptotic upper and lower bounds to $\mu_{i}^{h}$. For this purpose, let us remark that according to the min--max-principle (max-min-principle, respectively) we have

$$
\begin{gathered}
\mu_{i}^{h}=\min _{\mathscr{H}_{i}^{h} \subset W\left(Q_{\lambda}^{h}\right)} \max _{\Phi \in \mathscr{H}_{i}^{h}} \hat{\mathscr{R}}(\Phi) \\
=\max _{\mathscr{H}_{v-i+1}^{h} \subset W\left(Q_{\lambda}^{h}\right)} \min _{\Phi \in \mathscr{H}_{v-i+1}^{h}} \hat{\mathscr{R}}(\Phi) .
\end{gathered}
$$

Using Theorem 4.2 we find

$$
\left\|P_{ \pm \omega} \Phi\right\| \leqq c \lambda^{2} h^{2}\left\|P_{ \pm \omega} \Phi\right\|+\left\|Q_{\lambda}^{h} P_{ \pm \omega} \Phi\right\| \text {. }
$$

Thus for sufficiently small $h$

$$
\left\|P_{ \pm \omega} \Phi\right\| \leqq c\left\|Q_{\lambda}^{h} P_{ \pm \omega} \Phi\right\| .
$$

Let $B: \mathscr{H}_{*, s} \rightarrow \mathscr{H}_{*, s}, \Phi \rightarrow B \Phi$, be an operator defined by

It holds that

$$
(\psi, B \Phi)_{*, s}=(M \psi, \Phi)-(\psi, M \Phi) \text { for all } \psi \in \mathscr{H}_{*, s} .
$$

$$
\left\|P^{h} B \Phi\right\|+h^{1 / 2}\left\|P^{h} B \Phi\right\|_{*, s} \leqq c h^{2}\|\Phi\|
$$

for sufficiently small $h$ (see [10], Section 3).

Since

$$
\operatorname{Re}\left(P_{-\omega} U, \mathscr{M} P_{-\omega} U\right)=-\omega\left\|P_{-\omega} U\right\|^{2},
$$

we obtain, using in turn Theorem 4.2, (4.24) and inequalities (4.25)-(4.27) for $\Phi=Q_{\lambda}^{h} P_{-\omega} U$

$$
\begin{aligned}
& \operatorname{Re}(\Phi, \mathscr{M} \Phi) \\
& =\operatorname{Re}\left(P_{-\omega} U, \mathscr{M} P_{-\omega} U\right)+\operatorname{Re}\left(\Phi-P_{-\omega} U, \mathscr{M}\left(\Phi-P_{-\omega} U\right)\right) \\
& +\operatorname{Re}\left(P_{-\omega} U, \mathscr{M}\left(\Phi-P_{-\omega} U\right)\right)+\operatorname{Re}\left(\Phi-P_{-\omega} U, \mathscr{M}_{-\omega} P_{-\omega} U\right) \\
& =-\omega\|\Phi\|^{2}+\mathcal{O}\left(h^{3 / 2}\right)\|\Phi\|^{2}, \quad h \rightarrow 0 .
\end{aligned}
$$

In a similar way, it can be proved that

$$
\operatorname{Re}(\Phi, \mathscr{M} \Phi)=\omega\|\Phi\|^{2}+\mathcal{O}\left(h^{3 / 2}\right)\|\Phi\|^{2}, \quad h \rightarrow 0, \text { for all } \Phi=Q_{\lambda}^{h} P_{\omega} U .
$$

By the min-max-principle and by (4.28) we obtain

$$
\mu_{i}^{h} \leqq \max _{\Phi \in W\left(Q_{\lambda}^{h} P_{-}\right)} \hat{\mathscr{R}}(\Phi)=-\omega+\mathcal{O}\left(h^{3 / 2}\right), \quad h \rightarrow 0,
$$


for $i=v / 2+1, \ldots, v$ and, respectively, by the max-min-principle and by (4.29)

$$
\mu_{i}^{h} \geqq \min _{\Phi \in W\left(Q_{\lambda}^{h} P_{\omega}\right)} \hat{\mathscr{R}}(\Phi)=\omega+\mathcal{O}\left(h^{3 / 2}\right), \quad h \rightarrow 0,
$$

for $i=1, \ldots, v / 2$.

As a corollary to Theorem 4.2 and to estimates (4.30) and (4.31) we can prove

Theorem 4.3. The asymptotic error estimate

$$
\left\|P_{\omega} U-P_{\omega}^{h} P_{\omega} U\right\|_{*, s} \leqq c\left(\omega_{0}\right) h^{3 / 2}\left\|P_{\omega} U\right\|
$$

holds for all $-\omega_{0} \leqq \omega \leqq \omega_{0}$.

Proof. The case $\omega=0$ is trivial. At first let $\omega>0$. Using decomposition (4.18) and Theorem 4.2 we find

$$
\left\|P_{\omega} U-P_{\omega}^{h} P_{\omega} U\right\|_{*, s} \leqq c\left(\omega_{0}\right) h^{2}\left\|P_{\omega} U\right\|_{*, s}+\left\|P_{-\omega}^{h} P_{\omega} U\right\|_{*, s} .
$$

Since $(\mathscr{M}+\omega) P_{\omega} U=2 \omega P_{\omega} U$, we obtain by (4.23) and by estimate (4.27)

$$
\begin{aligned}
& \left\|P_{-\omega}^{h} P_{\omega} U\right\|^{2} \\
& \leqq \frac{1}{(2 \omega)^{2}} \sum_{i=v / 2+1}^{v}\left((\mathscr{M}+\omega) P_{\omega} U, \Phi_{i}^{h}\right)^{2} \\
& \leqq \frac{1}{(2 \omega)^{2}}\left\|P_{\omega} U\right\|^{2}\left(\sum_{i=v / 2+1}^{v}\left\|(\mathscr{M}+\omega) \Phi_{i}^{h}\right\|^{2}+c h^{3}\left\|P_{\omega} U\right\|_{*, s}^{2}\right) .
\end{aligned}
$$

Using (4.30) we conclude for $\Phi_{i}^{h} \in W\left(Q_{\lambda}^{h}\right), i=v / 2, \ldots, v$,

$$
\begin{aligned}
& \left\|(\mathscr{M}+\omega) \Phi_{i}^{h}\right\|^{2} \\
& =\left\|\mathscr{M} \Phi_{i}^{h}\right\|^{2}+2 \omega \mu_{i}^{h}\left\|\Phi_{i}^{h}\right\|^{2}+\omega^{2}\left\|\Phi_{i}^{h}\right\|^{2} \\
& \leqq\left\|\mathscr{M} \Phi_{i}^{h}\right\|+\left(\mathcal{O}\left(h^{3 / 2}\right)-\omega^{2}\right)\left\|\Phi_{i}^{h}\right\|^{2}, \quad h \rightarrow 0 .
\end{aligned}
$$

Since by (2.9) for all $\Phi \in \mathscr{H}$

$$
\left\|Q_{\lambda}^{h} \Phi\right\|_{*, s}^{2} \leqq \lambda^{h}\left\|Q_{\lambda}^{h} \Phi\right\|^{2}
$$

and since $\lambda^{h} \leqq \omega^{2}+1+c \hat{\lambda}^{2} h^{2}$, we obtain

$$
\left\|\mathscr{M} Q_{\lambda}^{h} \Phi\right\|^{2}+\left\|Q_{\lambda} \Phi\right\|^{2} \leqq\left(\omega^{2}+1+\mathcal{O}\left(h^{2}\right)\right)\left\|Q_{\lambda}^{h} \Phi\right\|^{2}, \quad h \rightarrow 0 .
$$

Moreover, recalling that $\operatorname{div} \varepsilon\left(Q_{\lambda} U\right)_{1}=0$, we have by Theorem 4.1

$$
\left\|\operatorname{div}\left(Q_{\lambda}^{h} \Phi\right)_{1}\right\|^{2}=\mathcal{O}\left(h^{2}\right)\left\|\Phi_{i}^{h}\right\|^{2}, \quad h \rightarrow 0 .
$$

Accordingly, by (4.35) and (4.36),

$$
\left\|(\mathscr{M}+\omega) \Phi_{i}^{h}\right\|^{2}=\mathcal{O}\left(h^{3 / 2}\right)\left\|\Phi_{i}^{h}\right\|^{2}, \quad h \rightarrow 0 .
$$

Hence, by (4.34)

$$
\left\|P_{-\omega}^{h} P_{\omega}^{h} U\right\|^{2}=\mathcal{O}\left(h^{3 / 2}\right)\left\|P_{\omega} U\right\|^{2} .
$$


Using the same arguments as in the deduction for inequality (4.34) together with (4.37) we obtain

$$
\left\|\mathscr{M} P_{-\omega}^{h} P_{\omega} U\right\|^{2}=\mathcal{O}\left(h^{3 / 2}\right)\left\|P_{\omega} U\right\|^{2}\left\|\Phi_{i}^{h}\right\|^{2} .
$$

If we combine (4.33), (4.37), (4.39) and (4.40), the assertion of Theorem 4.3 follows for $\omega>0$.

The case $\omega<0$ follows in the same way if we use (4.31) instead of (4.30).

Acknowledgements. This paper is supported by Sonderforschungsbereich 72 at the University of Bonn, which is sponsored by the Deutsche Forschungsgemeinschaft. The first author is pleased to thank Professor R. Leis and all members of the Department of Applied Mathematics for stimulating discussions and for their warm hospitality during the author's stay at the University of Bonn in 1978-1979.

\section{References}

[1] CANuto, C.: Eigenvalue approximations by mixed methods. - RAIRO Anal. Numér. 12, 1978, 27-50.

[2] Descloux, J., N. Nassif, and J. Rappaz: On spectral approximation. Part 1. The problem of convergence. - RAIRO Anal. Numér. 12, 1978, 97-112.

[3] Descloux, J., N. Nassif, and J. Rappaz: On spectral approximation. Part 2. Error estimates for Galerkin method. - RAIRO Anal. Numér. 12, 1978, 113-119.

[4] FIX, G. J.: Eigenvalue approximation by finite element method. - Adv. in Math. 10, 1973, $300-316$.

[5] Ishihara, K.: Convergence of the finite element method applied to the eigenvalue problem $\Delta u+\lambda u=0$. - Publ. Res. Inst. Math. Sci. 13, 1977, 47-60.

[6] Ishihara, K.: A mixed finite element method for the biharmonic eigenvalue problems of plate bending. - Publ. Res. Inst. Math. Sci. 14, 1978, 399-414.

[7] KIKUCHI, F.: On the finite element scheme based on the discrete Kirchhoff assumption. - Numer. Math. 24, 1975, 211-231.

[8] LeIs, R.: Zur Theorie der zeitungabhängigen Maxwellschen Gleichungen. - Berichte der Gesellschaft für Mathematik und Datenverarbeitung Bonn 50, St. Augustin, 1971.

[9] LeIs, R.: Rand- und Eigenwertaufgaben in der Theorie elektromagnetischer Schwingungen. Methoden und Verfahren der mathematischen Physik 11, Bibliographisches Inst. Mannheim, 1974, 88-116.

[10] NeittaAnmäKI, P., and R. PicARD: Error estimates for the finite element approximation to a Maxwell-type boundary value problem. - Numer. Funct. Anal. Optim. 2, 1980, $263-285$.

[11] Neittaanmäki, P., and R. Picard: On the finite element method for time-harmonic acoustic boundary value problems. - Comput. Math. Appl. 7, 1981, 127-138.

[12] Neittaanmäki, P., and J. Saranen: On the finite element approximation for Maxwell's problem in polygonal domains of plane. - Applicable Anal. 12, 1981, 73-83.

[13] OBsBorn, J. E.: Spectral approximation for compact operators. - Math. Comp. 23, 1975, $712-725$.

[14] PICARD, R.: Zur Theorie der zeitabhängigen Maxwellschen Gleichungen mit Randbedingungen $n(\nabla \times E)=n(\nabla \times H)=0$ im inhomogenen anisotropen Medium. - Bonner Math Schr. 65, 1973. 
[15] PICARD, R.: Ein Randwerproblem für die zeitabhängigen Maxwellschen Gleichungen mit Randbedingungen $n \varepsilon E=n \mu H=0$ in beschränkten Gebieten beliebigen Zusammenhangs. - Applicable Anal. 6, 1977, 207-221.

[16] RANNACHER, R.: Nonconforming finite element methods for eigenvalue problems of linear plate theory. - Numer. Math. 33, 1979, 23-42.

[17] Saranen, J.: Über die Approximation der Lösungen der Maxwellschen Randwertaufgabe mit der Methode der finite Elemente. - Applicable Anal. 10, 1980, 15-30.

[18] Saranen, J.: Finite element method for the electric field of Maxwell's boundary value problem in polygonal domains of plane. - Ann. Acad. Sci. Fenn. Ser. A I Math. 6, 1981 (to appear).

[19] Strang, G., and G. J. Fix: An analysis of the finite element method. - Prentice-Hall, Englewood Cliffs, New York, 1973.

[20] Stummel, F.: Approximation methods for eigenvalue problems in elliptic differential equations. - Proc. Conf. Numerik und Anwendungen von Eigenwertaufgaben und Verzweigungsproblemen (Oberwolfach 1976), E. Bohl, L. Collatz, K. P. Hadeler, ed., Birkhäuser, Basel, ISNM 38, 1977, 133-165.

[21] Neittaanmäki, P., and J. Saranen: Finite element approximation of electromagnetic fields in the three dimensional space. - Numer. Funct. Anal. Optim. 2, 1980, 487-506.

University of Jyväskylä

Department of Mathematics

Sammonkatu 6

SF-40100 Jyväskylä 10

Finland
University of Bonn

Department of Applied Mathematics

Wegelerstrasse 10

D-5300 Bonn 1

Federal Republic of Germany

Received 6 March 1981 